\title{
Detailed Balance and Equilibrium
}

\author{
A. Verbeure \\ Instituut voor Theoretische Natuurkunde, Universiteit Leuven, B-3030 Leuven, Belgium
}

\begin{abstract}
For classical lattice systems, an infinite set of jump-processes satisfying the condition of detailed balance is found. It is proved that any state invariant for these processes is an equilibrium state, providing a new characterization of DLR-states by means of the notion of detailed balance. This extends previous results, proved in one and two dimensions.
\end{abstract}

\section{Introduction}

Since their first appearance $[1,2]$ in rigorous statistical mechanics, the equilibrium conditions, known as the DLR-conditions, have been reformulated in various alternative ways. Without being exhaustive at all, one has proved that the translation invariant DLR-states minimize the free energy [3], that they are characterized by an inequality expressing a balance between energy and entropy [4], etc. ... We want to add another characterization and this by means of the notion of detailed balance which is widely used in the physics literature $[5,6]$.

The detailed balance condition has a well-defined physical meaning, it expresses the duality of a jump process between two states with its inverse process.

This notion entered the mathematics literature under the name of a reversible process $[7,8]$. We will continue to use the name detailed balance and give an independent definition. Our definition will also turn out to have a more universal character than the previous one.

Our main result is that we are able to construct explicitly an infinite set of detailed balance processes, and that we prove that any state which is invariant for all these processes is necessarily a DLR-state, even when the state or the potential is not translation invariant. This extends earlier results (see discussion). We also want to stress the extreme simplicity of the proof.

\section{Detailed Balance Processes and DLR-Conditions}

Consider the lattice $Z^{v}(v=1,2,3, \ldots)$. To each site $j \in Z^{v}$ we associate a copy $K_{j}$ of a compact set $K$. For any subset $X \subset Z^{v}$, denote $K_{X}=\prod_{j \in X} K_{j}$ and $C(X)$ the set of real 
valued continuous functions on $K_{X}$; the set of continuous functions on $\Omega=K_{Z}$ is denoted by $C(\Omega)$, it is the sup-norm closure of $\bigcup_{X \subset Z^{v}} C(X)$.

Let us first recall a version of the DLR-conditions which is less known $[7,9]$.

Denote by $Q$ the group of transformations of $C(\Omega)$, the transposed of the invertible transformations of the configuration space $\Omega$, changing the configurations in some fixed finite volume only (it contains single spin flips in Ising models, spin exchanges, etc.).

A map $h$ from $Q$ into $C(\Omega)$ is called a relative Hamiltonian if for any pair $\tau_{1}, \tau_{2} \in Q$ holds

$$
h\left(\tau_{1} \tau_{2}\right)=\tau_{1} h\left(\tau_{2}\right)+h\left(\tau_{1}\right) .
$$

For a given relative Hamiltonian $h$ there might exist a family of local Hamiltonians $H_{\Lambda} \in C(\Lambda), \Lambda \subset Z^{v}$, such that $h(\tau)=\lim _{\Lambda} \tau H_{\Lambda}-H_{\Lambda}$. Therefore the relative Hamiltonian represents the energy difference of any configuration with its transformed one under the map $\tau$. However in what follows we do not need the existence of Hamiltonians.

A classical dynamical lattice system is given by its algebra of observables $C(\Omega)$ and by a relative Hamiltonian $h$. Such a system might have an equilibrium state at a certain inverse temperature $\beta(>0)$. The latter is defined by

Definition II.1. A state $\omega_{\beta}$ of $C(\Omega)$ satisfies the DLR-conditions at inverse temperature $\beta>0$, with a relative Hamiltonian $h$ if for all $f \in C(\Omega)$ and $\tau \in Q$ holds:

$$
\omega_{\beta}\left(\tau^{-1}(f)\right)=\omega_{\beta}(f \exp (-\beta h(\tau))) .
$$

In fact it is sufficient to have this condition for a set of elements $\tau \in Q$ separating the points of $Q$.

Now we are in a position to define what we mean by an evolution satisfying the detailed balance condition.

Definition II.2. Let $\left(\gamma_{t}\right)_{t \in \mathbb{R}}$ be a one-parameter strongly continuous semigroup of unity preserving positive maps of $C(\Omega)$. Then it is said to satisfy the detailed balance condition at $\beta$ if for all $f, g \in C(\Omega)$ and for all DLR-states $\omega_{\beta}$ holds

$$
\omega_{\beta}\left(f \gamma_{t} g\right)=\omega_{\beta}\left(\gamma_{t}(f) g\right) .
$$

This definition is the classical analogue of the one developed in [10] for quantum systems. It differs from other definitions of detailed balance $[7,8,11]$ in the sense that it is not a condition on a state but only on the dynamics for a given dynamical system $(C(\Omega), h)$. Therefore our definition has a more universal character.

Now we are looking for solutions of Eq. (2). For any $\tau \in Q$, consider the following linear map $L_{\tau}$ of $C(\Omega)$ into $C(\Omega)$ :

$$
L_{\tau}=e^{-\frac{\beta h(\tau)}{2}}(\tau-1)+e^{-\frac{\beta h(\tau-1)}{2}}\left(\tau^{-1}-1\right) .
$$

One readily checks that

$$
L_{\tau}(1)=0 \text {, }
$$


and that for all $f \in C(\Omega)$

$$
\begin{gathered}
L_{\tau}\left(f^{2}\right)-2 f L_{\tau}(f)=e^{-\frac{\beta h(\tau)}{2}}((\tau-1) f)^{2}+e^{-\frac{\beta h\left(\tau^{-1}\right)}{2}}\left(\left(\tau^{-1}-1\right) f\right)^{2} \geqq 0, \\
\left\|L_{\tau}(f)\right\| \leqq 4\|f\| \exp (\beta\|h(\tau)\| / 2) .
\end{gathered}
$$

Because of (6) the map $L_{\tau}$ is exponentiable and is the generator of a semigroup

$$
\gamma_{t}^{\tau}=\exp t L_{\tau} ; t \geqq 0
$$

From (4) one gets $\gamma_{t}^{\tau}(1)=1$, and together with (5) it follows that $\gamma_{t}^{\tau}$ is a strongly continuous semigroup of unity preserving, positive contraction maps. One has also:

Proposition II.3. For any $\tau \in Q$, the semigroup $\left(\gamma_{t}^{\tau}\right)_{t \geqq 0}$ defined in (7) satisfies the detailed balance condition at $\beta$.

Proof. From (2) and (7) it is clearly sufficient to prove for all $f, g \in C(\Omega)$, and $\omega_{\beta}$ a DLR-state that

$$
\omega_{\beta}\left(g L_{\tau} f\right)=\omega_{\beta}\left(L_{\tau}(g) f\right) .
$$

Now

$\omega_{\beta}\left(g L_{\tau} f\right)=-\omega_{\beta}\left(\left(e^{-\frac{\beta}{2} h(\tau)}+e^{-\frac{\beta}{2} h\left(\tau^{-1}\right)}\right) g f\right)+\omega_{\beta}\left(e^{-\frac{\beta}{2} h(\tau)} g \tau f+e^{-\frac{\beta}{2} h\left(\tau^{-1}\right)} g \tau^{-1} f\right)$.

But using the property

$$
\tau^{-1} h(\tau)+h\left(\tau^{-1}\right)=0
$$

which follows from (1), and using the DLR-condition, one obtains subsequently

$$
\begin{aligned}
\omega_{\beta}\left(e^{-\frac{\beta}{2} h(\tau)} g \tau f\right) & =\omega_{\beta}\left(\tau\left(e^{-\frac{\beta}{2} \tau^{-1} h(\tau)} \tau^{-1}(g) f\right)\right) \\
& =\omega_{\beta}\left(e^{\frac{\beta}{2} h\left(\tau^{-1}\right)} \tau^{-1}(g) f e^{-\beta h\left(\tau^{-1}\right)}\right) \\
& =\omega_{\beta}\left(e^{-\frac{\beta}{2} h\left(\tau^{-1}\right)} \tau^{-1}(g) f\right),
\end{aligned}
$$

and analogously for the other term. After substitution in (9) one gets (8).

This proves that we have obtained a large set $\Gamma(h, \beta)=\left\{\gamma_{t}^{\tau} \mid \tau \in Q\right\}$ of dynamical semigroups satisfying the detailed balance condition. Their explicit form is given by expression (3). It is interesting to remark that indeed they do correspond to a jump process, the jump described by $\tau$, such that the probability for that jump is proportional to the Gibbs factor. Furthermore the symmetry between the jump $\tau$ and its inverse is clear.

One can also construct a set of processes which are space translation invariant. Therefore take any element of the type defined by formula (3) and add to it all its space translates. Then one proves as in [12] that it is the generator of a dynamical semigroup on $C(\Omega)$ (see also [13]). 
Let us now come to our main result. We prove that the set $\Gamma(h, \beta)$ of solutions of Eq. (3) is large enough to characterize equilibrium states. We suppose that the states under consideration are absolutely continuous with respect to an a priori given measure $\varrho_{0}$, and assume that the set $\left\{\tau \in Q \mid \tau-1\right.$ invertible on $\left.L^{1}\left(\varrho_{0}, \Omega\right)\right\}$ separates the points of $\Omega$.

Theorem II.4. Let $\omega$ be any state of $C(\Omega)$ such that $\omega \cdot \gamma_{t}^{\tau}=\omega$ for all $t \geqq 0$ and for all $\gamma_{t}^{\tau} \in \Gamma(h, \beta)$. Then $\omega$ is a DLR-state at inverse temperature $\beta$ and with relative Hamiltonian $h$.

Proof. Clearly $\omega \cdot \gamma_{t}^{\tau}=\omega$ for all $\tau \in Q$, and $t \in \mathbb{R}^{+}$, yields $\omega \cdot L_{\tau}=0$ for all $\tau \in Q$. As

$$
h\left(\tau^{-1}\right)=-\tau^{-1} h(\tau), e^{-\frac{\beta}{2} h\left(\tau^{-1}\right)}\left(\tau^{-1}-1\right)=-\tau^{-1}\left(e^{\frac{\beta}{2} h(\tau)}(\tau-1)\right),
$$

and so for all $f \in C(\Omega)$ :

$$
0=\omega\left(L_{\tau} f\right)=\omega\left(e^{-\frac{\beta}{2} h(\tau)}(\tau-1) f-\tau^{-1}\left(e^{\frac{\beta}{2} h(\tau)}(\tau-1) f\right)\right) .
$$

Hence

$$
0=\omega\left(\left(e^{-\beta h(\tau)}-\tau^{-1}\right)\left(e^{\frac{\beta}{2} h(\tau)}(\tau-1) f\right)\right) .
$$

But this is the DLR-condition for functions of the type

$$
e^{\frac{\beta}{2} h(\tau)}(\tau-1) f, f \in C(\Omega) .
$$

This set is dense in $C(\Omega)$. Hence we have the DLR-equation for a set transformations separating the points in $\Omega$. This proves the theorem.

\section{Discussion}

By the theorem we realized a characterization of the equilibrium states as those states which are invariant under the processes $\Gamma(h, \beta)$, satisfying the detailed balance condition. Here one might refer to other work in this direction. Although by a completely different approach, the lattice translation invariant DLR-states are characterized by the detailed balance processes before (see e.g. [7], Theorem 3.42 and Corollary $3.44 ;$ [14]). Finally, in one and two dimensions, the (not necessarily) translation invariant Gibbs states are also characterized as the stationary measures for a stochastic process in [15]. All these results are based on free energy considerations. Hence apart from the new way of looking at the condition of detailed balance our main contribution consists in the characterization of all DLR-states, including the non-translation invariant ones and in any dimensions.

Acknowledgements. The author thanks P. Vanheuverzwijn for pointing out [15].

\section{References}

1. Dobrushin, R.L.: Theory Probab. Appl. 13, 197-224 (1968)

2. Lanford, O.E., Ruelle, D.: Observables at infinity and states with short range correlations in statistical mechanics. Commun. Math. Phys. 13, 194-215 (1969) 
3. Ruelle, D.: A variational formulation of equilibrium statistical mechanics and the Gibbs phase rule. Commun. Math. Phys. 5, 324 (1967)

4. Fannes, M., Vanheuverzwijn, P., Verbeure, A.: Energy-entropy inequalities for classical lattice systems. J. Stat. Phys. 29, 547-558 (1982)

5. Agarwal, G.S.: Open quantum Markovian systems and microreversibility. Z. Physik 258, 409 (1973)

6. Carmichael, H.J., Walls, D.F.: Detailed balance in open quantum Markoffian systems. Z. Phys. B-Condensed Matter and Quanta 23, 299 (1976)

7. Georgii, H.O.: Canonical Gibbs measures. In: Lecture Notes in Mathematics, Vol. 760. Berlin, Heidelberg, New York: Springer 1979

8. Sullivan, W.G.: Markov processes for Random fields. Comm. Dublin Institute for Advanced Studies. Series A, No. 23

9. Fannes, M., Verbeure, A.: On solvable models in classical lattice systems. Commun. Math. Phys. (to appear)

10. Quagebeur, J., Stragier, G., Verbeure, A.: Ann. Inst. H. Poincaré (to appear)

11. Kossakowski, A., Frigerio, A., Gorini, V., Verri, M.: Quantum detailed balance and KMS condition. Commun. Math. Phys. 57, 97 (1977)

12. Robinson, D.W.: Statistical mechanics of quantum spin systems. II. Commun. Math. Phys. 7, 337 (1968)

13. Liggett, Th.M.: Trans. Am. Math. Soc. 165, 471 (1971)

14. Holley, R.A.: Free energy in a Markovian model of a lattice spin system. Commun. Math. Phys. 23, 87 (1971)

15. Holley, R.A., Stroock, D.W.: In one and two dimensions, every stationary measure for a stochastic Ising model is a Gibbs state. Commun. Math. Phys. 55, 37 (1977)

Communicated by H. Araki

Received April 4, 1984 
